\title{
Breakdown Phenomena across Micrometer Scale Surface Gap under Negative Voltage Application
}

\author{
Hiroyuki Iwabuchi and Tsutomu Oyama \\ Yokohama National University \\ Faculty of Engineering \\ 79-5 Tokiwadai, Hodogaya-ku, Yokohama, 240-8501 Japan
}

\author{
Akiko Kumada and Kunihiko Hidaka \\ The University of Tokyo \\ Department of Engineering and Information Systems \\ 7-3-1 Hongo, Bunkyo-ku, Tokyo, 113-8656 Japan
}

\begin{abstract}
With the miniaturization of electrical devices, the insulation width and the separation between electrodes have been accordingly reduced. Consequently, electrical breakdown across micrometer-scale gaps is of great practical interest for insulation design. In this paper, electrical breakdown across micrometer scale surface gap in atmospheric air was investigated under inhomogeneous field. The breakdown was measured under negative impulse voltage and the generation and movement of neutral and charged particles were simulated based on PIC-MCC (particle-in-cell with Monte Carlo collision). The result shows that the breakdown phenomena across micrometer scale gaps is mainly determined by field emission current from the cathode surface, similar to the discharge process in vacuum. The positive ions generated by collision of electrons to neutral particles enhance the electric field near the cathode, resulting in the increase of the field emission current from the cathode.
\end{abstract}

Index Terms - electrical devices, micrometer-scale gap, vacuum breakdown, electric field emission, PIC-MCC method

\section{INTRODUCTION}

IN recent years, the development of semiconductor manufacturing technology has accelerated the miniaturization of electrical and MEMS devices. The application of these device has been widely increased [1,2]. For example, electrostatic actuation with low power consumption and simple design has been developed as an efficient method based on MEMS technology. For efficient operation of these actuators, a large force between driving electrodes with micrometer-scale separation is required. Because the force is proportional to the square of the electric field strength, the most convenient method to raise the force can be attained by making the gap smaller which means narrowing the electrode separation [3]. By narrowing the insulation thickness, these devices are exposed to high risk of breakdown which causes serious damage. Therefore, it is necessary to investigate the insulation strength of micrometer-scale gaps which are contained in such devices [3-7].

Earlier studies suggested that the breakdown characteristics across such a small gap were different from the one across $\mathrm{cm}$ scale

Manuscript received on 9 November 2018, in final form 28 March 2019, accepted xx Month 20yy. Corresponding author: H. Iwabuchi. gaps [8,9]. A modified Paschen's curve was also introduced [1012] in order to explain the breakdown characteristics under spheresphere electrode configuration. Recently, research on breakdown characteristics across such a small gap has been widely reported [13-15], however, it is still a great challenge to clarify the detail mechanism of breakdown phenomena across micrometer scale surface gap.

We have measured the breakdown and the pre-breakdown characteristics across aluminum surface gaps $[16,17]$ and it was found that the breakdown phenomena across micrometer scale gaps resemble the breakdown phenomena under vacuum. The result can be explained by comparing the mean free path of electrons and the gap width. However, these reports do not really represent the situation in the breakdown phenomena across micrometer surface gap considering the electric field emission from the cathode surface. In this paper, the breakdown phenomena in micrometer scale surface gaps with tungsten electrodes were experimentally investigated changing the gap width. Breakdown voltage and the light emission were simultaneously observed under negative impulse voltage and the particle simulation based on PICMCC (particle-in-cell with Monte Carlo collision) method was conducted considering the electric field emission. Breakdown process across micrometer scale gap was investigated through these results. 


\section{EXPERIMENTAL SETUP}

\subsection{MICROMETER-SCALE SURFACE GAP FABRICATED ON OXIDIZED-SILICON WAFER}

A pair of metal electrode was fabricated on $\mathrm{SiO}_{2}$ insulation layer by using semiconductor manufacturing technology as shown in Figure 1. The gap between electrodes was set at 1 to $5 \mu \mathrm{m}$. Oxidized silicon wafer was consisted of $525 \mu \mathrm{m}$ thick silicon and $2 \mu \mathrm{m}$ thick silicon dioxide. The silicon layer was $\mathrm{p}$ type semiconductor and its volume resistivity was $1-100 \Omega \mathrm{cm}$. On the top layer, tungsten electrodes of $100 \mathrm{~nm}$ thickness were fabricated. In this study, Si layer was grounded when the voltage was applied.

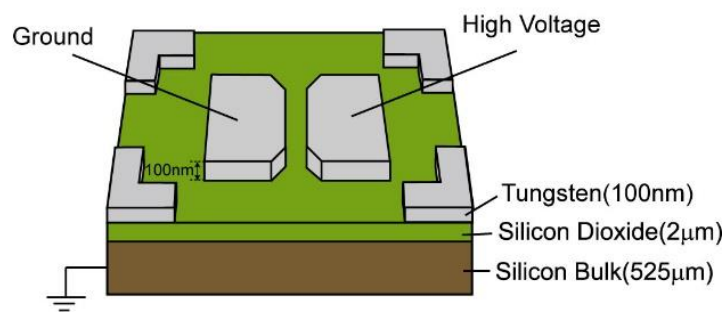

(a) Bird's eye view

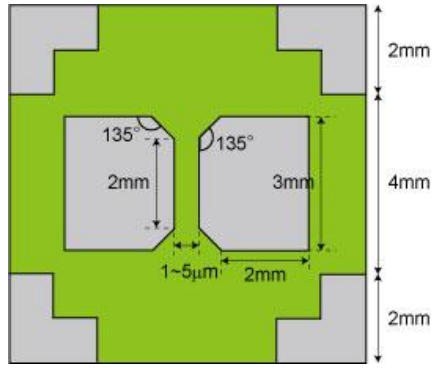

(b) Top-view

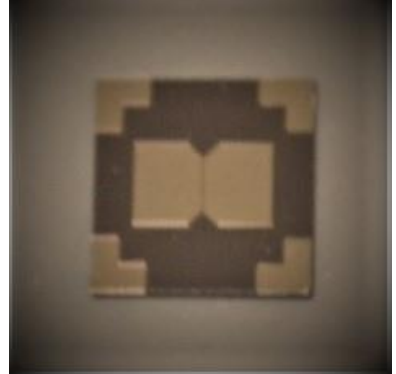

(c) Photograph of the sample
Figure 1. Micrometer-scale surface gap on silicon-oxide wafer. A pair of tungsten electrode was fabricated on $\mathrm{SiO}_{2}$ insulation layer and the gap between electrodes was set at 1 to $5 \mu \mathrm{m}$. The surface roughness of the sample was measured by Fowler-Nordheim plot shown in Section 3.3. The measured field enhancement factor was approximately 40 .

\subsection{MEASUREMENT CURCUIT}

The measurement circuit used in this study is shown in Figure 2. The high speed transistor switch (BEHLKE Power Electronics GmbH, HTS41) was utilized in the circuit to generate impulse voltage. This switch can be controlled by TTL signal to set the on and off time. The capacitor was $1 \mathrm{nF}$ and current limiting resistance was $2 \mathrm{k} \Omega$ to prevent severe damage to the sample. The discharge current was measured by a current transformer (Tektronix CT1, $25 \mathrm{kHz}$ to $1 \mathrm{GHz}$ ). In order to minimize the effect of dust, all the experimental setups were put in a sealed cleanroom (class: 10000).

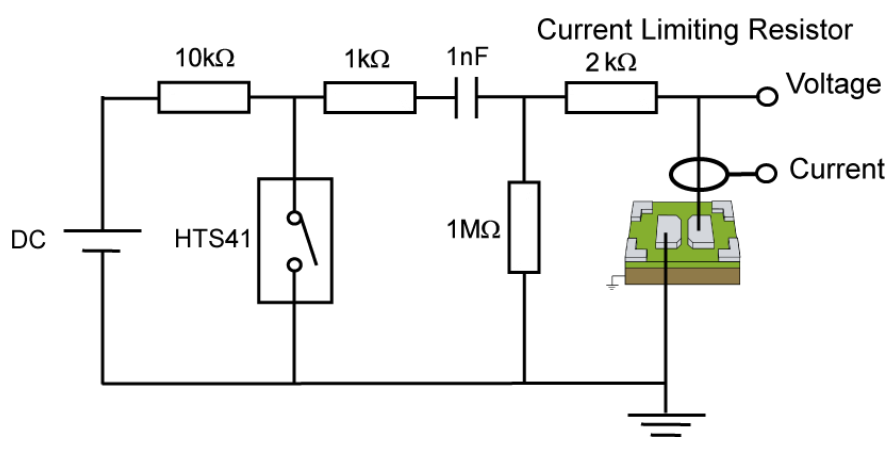

Figure 2. Measurement circuit used in this study. Negative impulse voltage was applied using high-speed transistor switch. Typical voltage and current waveforms are shown in Figure 4.

\subsection{ELECTRIC FIELD INTENSITY ACROSS MICROMETER-SCALE SURFACE GAP}

Electrostatic field distribution in the vicinity of micrometerscale surface gap was calculated based on finite element method using COMSOL Multiphysics Ver. 4.3b. The 3-D simulation model was constructed in this study. The volume resistivity of $\mathrm{SiO}_{2}$ was set at $10^{16} \Omega \mathrm{cm}$ and its relative permittivity was set at 3.9. The electric field intensities on the high-voltage electrode and the grounded electrode under application of DC $500 \mathrm{~V}$ are shown in Figure 3. The calculated point was set at 50-nm above the surface of the insulator on the tip of each electrode. The electric field intensity on the high voltage electrode is approximately $10^{9} \mathrm{~V} / \mathrm{m}$, which was independent of the gap width. On the other hand, the electric field intensity on the grounded electrode is strongly dependent on the gap width; the electric field intensity on the grounded electrode was under $10^{8}$ $\mathrm{V} / \mathrm{m}$ across 3 and $5 \mu \mathrm{m}$ gaps. The results can be explained by the effect of grounded layer $2 \mu \mathrm{m}$ below the high voltage electrode via $\mathrm{SiO}_{2}$ layer. The lines of electric force from the high voltage electrode mainly cross the insulator surface with this electrode configuration.

\section{EXPERIMENTAL RESULTS}

\subsection{VOLTAGE AND CURRENT WAVEFORMS}

Typical voltage and current waveforms when the discharge occurs are shown in Figure 4. The displacement current flows with the application of the impulse voltage at $t=0 \mu \mathrm{s}$. At $t=2.8$ $\mu \mathrm{s}$, the applied voltage sharply drops to $50 \mathrm{~V}$. The breakdown was determined by such drop of voltage and the light emission. Due to the voltage drop over current limiting resistance, the breakdown is unsustained and stops at $t=5.5 \mu \mathrm{s}$. The voltage started to recover and the second breakdown was induced. In this study, only the first breakdown is discussed as the breakdown across micrometer scale gap. 


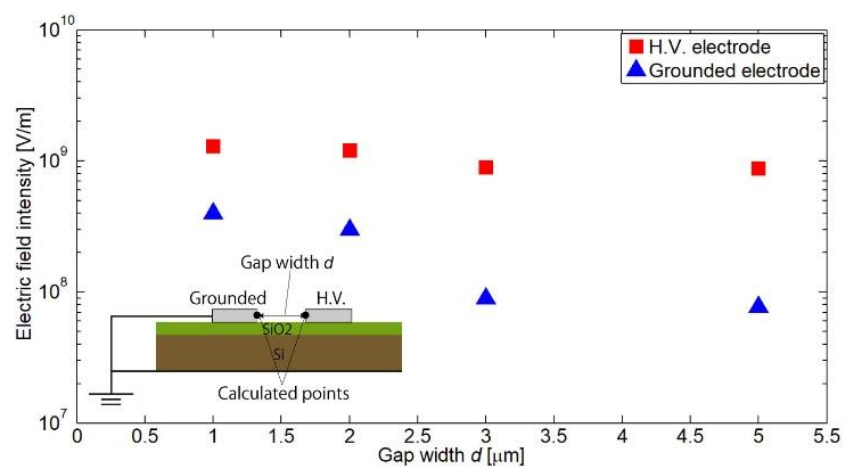

Figure 3. Electric field intensity on the calculated points on H.V. and grounded electrode. Red square and blue triangle symbols represent those at the tips on H.V. and grounded electrode, respectively. Because of the effect of conducting layer under the electrode, the electric field distribution was quite inhomogeneous.

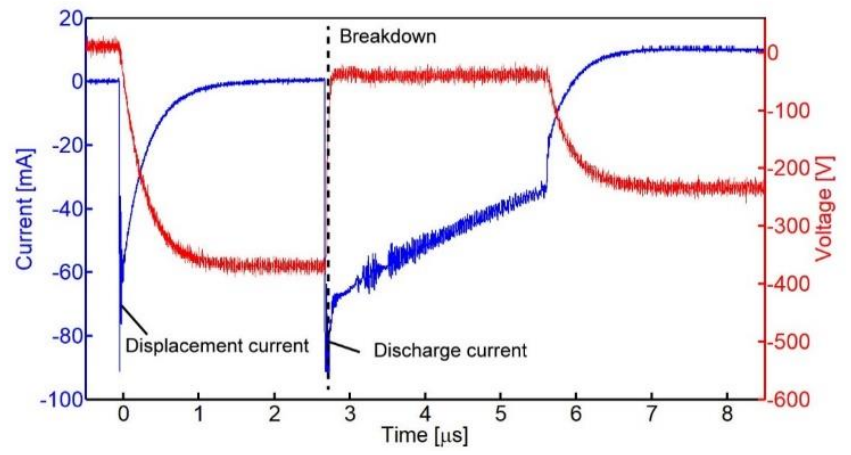

Figure 4. Typical voltage and current waveforms with occurrence of breakdown.

\subsection{BREAKDOWN CHARACTERISTICS ACROSS MICROMETER-SCALE SURFACE GAP}

Figure 5 shows the relationship between the gap width and the instantaneous voltage at the occurrence of breakdown under the negative impulse voltage application. The breakdown voltage was measured by increasing voltage from $50 \mathrm{~V}$ by $5 \mathrm{~V}$ on each sample. The experimental sample was replaced once the breakdown was observed. The result shows that the breakdown voltage has a variation between $100 \mathrm{~V}$ to $500 \mathrm{~V}$. It can be explained by the surface condition of the H.V. electrode. When the cathode surface is rough, the field emission current is easy to flow and induce breakdown. In this study, the condition of the electrode surface is expressed by field enhancement factor $\beta$.

Figure 6 represents the relationship between the number of voltage application and the breakdown voltage. Each point shown in Figure 6 shows the experimental result obtained in one sample across the $3 \mu \mathrm{m}$ gap. The figure clearly shows the conditioning effect: as the number of voltage application increases, the electrode surface becomes flat by field emission current and the breakdown voltage is higher.

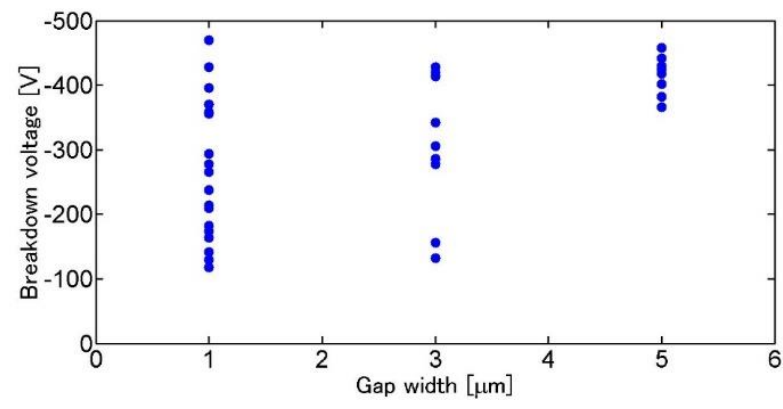

Figure 5. Relationship between gap width and the breakdown voltage.

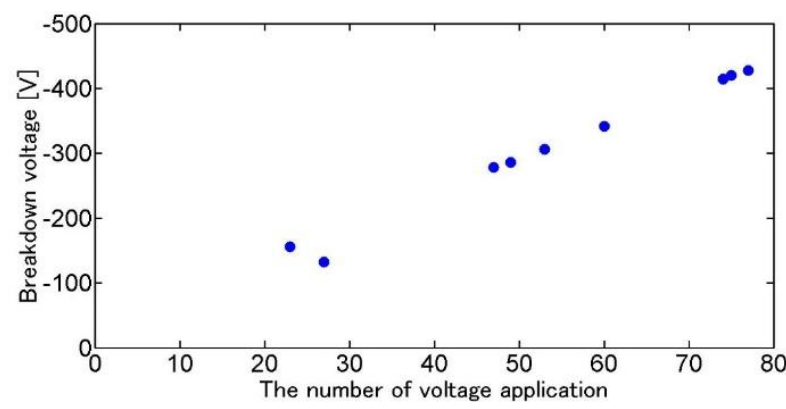

Figure 6. Relationship between the number of voltage application and breakdown voltage across the $3 \mu \mathrm{m}$ gap.

\subsection{MEASUREMENT OF FIELD ENHANCEMENT FACTOR}

The breakdown characteristics shows that the surface condition of the electrode can affect the breakdown voltage. In this study, the field enhancement factor $\beta$ was introduced to express the surface condition. The field enhancement factor $\beta$ was measured using the experimental circuit shown in Figure 7. The pulsed voltage was applied to the $1 \mu \mathrm{m}$ gap and the current was measured. After the displacement current flows, the DC field emission current (Fowler-Nordheim current) flows through the resistor. The DC current was measured using 5 samples changing the applied voltage and Fowler-Nordheim plot was obtained as shown in Figure 8. The result indicated that the field enhancement factor $\beta$ in this study was approximately 40 .

\subsection{DISCHARGE PATH ACROSS MICROMETER- SCALE SURFACE GAP}

The light emission from the electrodes with discharge occurrence was observed by ICCD camera (Andor, INSTASPEC V). In order to observe discharge path across micrometer-scale gap, the sample was set in a tilted position and the discharge path was observed at an angle shown in Figure 9. Typical light emission observed under negative impulse voltage application is shown in Figure 10. The discharge path was formed from the cathode to anode above the surface of the insulator. 


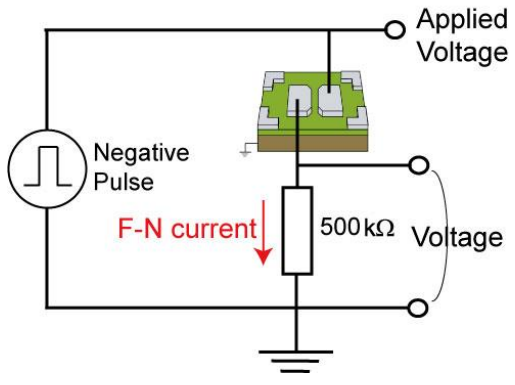

Figure 7. Measurement circuit for field enhancement factor.

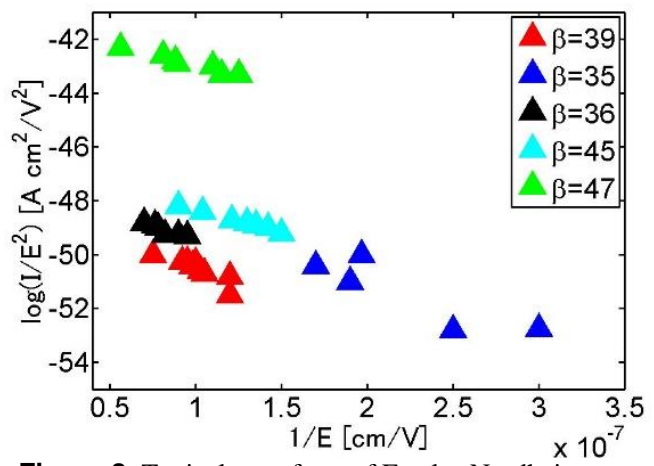

Figure 8. Typical waveform of Fowler-Nordheim current.

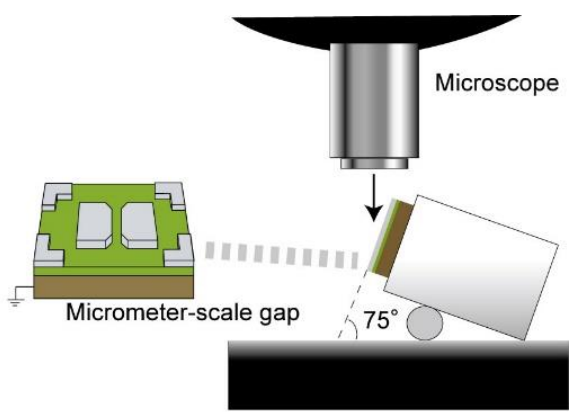

Figure 9. Observation of discharge path.

\section{BREAKDOWN SIMULATION BASED ON PIC-MCC METHOD}

\subsection{SIMULATION MODEL}

The breakdown process across micrometer-scale surface gap was simulated with PIC-MCC (Particle in Cell-Monte Carlo Collision) method using the software PEGASUS. The PICMCC tool calculates the breakdown processes basing on the Townsend criterion, therefore, photoionization phenomena are not considered. The simulation model is shown in Figure 11. The micrometer-scale gap was expressed in $x-y$ coordinate and the gas was consisted of $\mathrm{N}_{2}(80 \%)$ and $\mathrm{O}_{2}(20 \%)$ in atmospheric pressure. At $t=0 \mathrm{~s}, V=-100 \mathrm{~V}$ was applied to the H.V. electrode. The applied voltage corresponds to the measured breakdown voltage. The measuring point was introduced above the cathode tip in order to calculate the time dependence of ion density. The breakdown process was simulated including a flux of electrons at the cathode based on the Fowler-Nordheim theory [18] and the local electric field intensity. After emitting from the cathode, movement of electrons were simulated based on following collisions: electron-neutral collisions, i.e., elastic scattering, excitation, ionization to $\mathrm{N}_{2}^{+}$and $\mathrm{O}_{2}{ }^{+}$, and ion-neutral collisions, i.e., elastic scattering and charge exchange. The cross sections used in this study were obtained by Itikawa [19] and R. R. Laher [20]. On the insulator surface, charge accumulation and secondary electron emission were considered and the accumulated charge density was implemented in the calculation of the local electric field. In this simulation, $\beta=40$ was used as a field enhancement factor described in Section 3.3. The value of secondary electron emission coefficient on the insulator surface was set at 0.8 based on previous study [21].

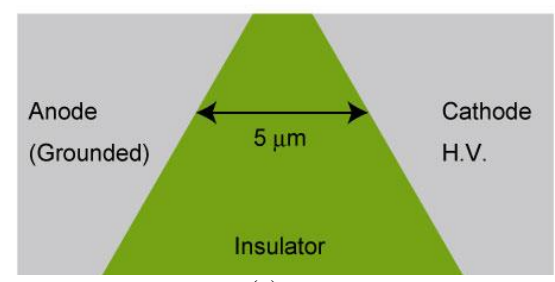

(a)

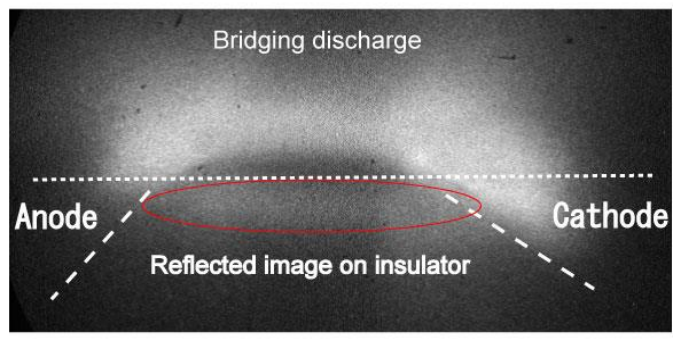

(b)

Figure 10. Typical discharge path observed under negative impulse voltage across $5 \mu \mathrm{m}$ gap. The discharge path was formed above the surface of the insulator under the negative voltage. (a) electrode configuration before the discharge (b) light emission when the discharge occurs

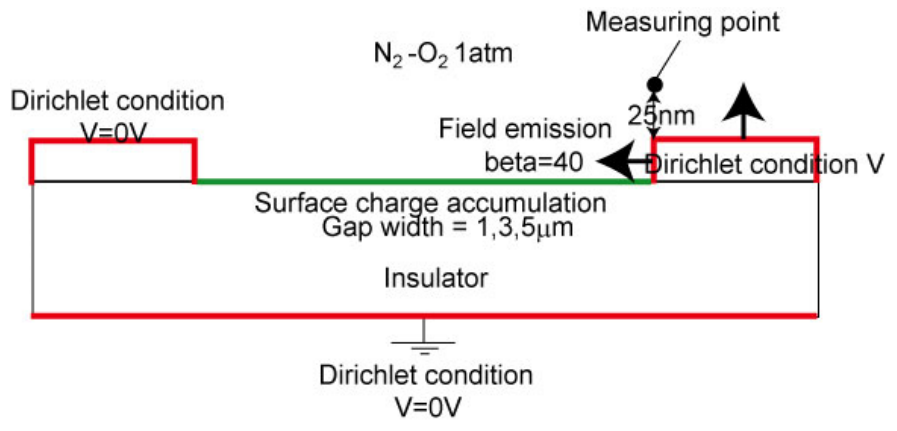

Figure 11. The simulation model demonstrating the micrometer-scale surface gap. The field emission current was considered on the cathode (the electrode on the right hand-side) surface. The surface condition was expressed by field enhancement factor $\beta$.

\subsection{SIMULATED RESULTS}

Figure 12 shows the time dependence of field emission current across 1,3 and $5 \mu \mathrm{m}$ gaps. The current was calculated 
as an integration of the number of electrons emitted from the H.V. electrode surface. As shown in the figure, field emission current is amplified when $-100 \mathrm{~V}$ was applied. On the other hand, under application of $-80 \mathrm{~V}$, field emission current is constant until $t=100 \mathrm{ps}$. The result shows that the breakdown was induced by field emission current and the breakdown voltage exists between $-80 \mathrm{~V}$ and $-100 \mathrm{~V}$. The result coincides well with the experimental one shown in Figure 5; by considering the field enhancement factor $\beta=40$, the breakdown voltage can decrease down to $-100 \mathrm{~V}$. As shown in Figure 12, the field emission current started to increase at $t=80 \mathrm{ps}$ in 5$\mu \mathrm{m}$ gap. That is why the breakdown voltage across $5-\mu \mathrm{m}$ gap is higher than the one across $3-\mu \mathrm{m}$ gap. Such a long voltage application can easily cause conditioning effect and field enhancement factor decreases. The low $\beta$, which indicates that the surface is flat, increases the breakdown voltage.

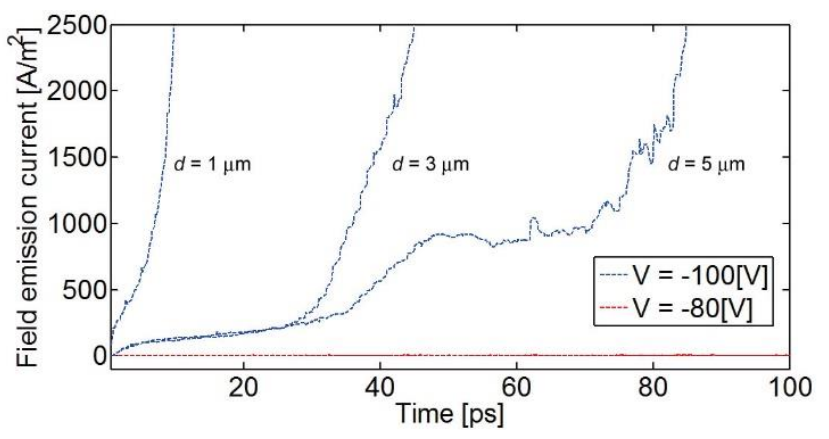

Figure 12. Amplification of Fowler-Nordheim current across the gap. The field emission current drastically increased when $-100 \mathrm{~V}$ was applied.

Figure 13 shows the time dependence of electron density across $1 \mu \mathrm{m}$ gap. The breakdown process is explained as follows; at (a) $t_{1}=1 \mathrm{ps}$, the electrons start to be emitted from the cathode subjected to Fowler-Nordheim equation. Electron density is relatively high in the vicinity of cathode. At (b) $t_{2}=5 \mathrm{ps,}$ emitted electrons bridge the gap and positive ions generated by electron-neutral collisions. From (c) $t_{3}=10 \mathrm{ps}$ to (d) $t_{4}=18 \mathrm{ps,}$ generated positive ions strengthen the electric field intensity on the cathode surface and field emission current increases. It causes the electron density between the gap to increase substantially. This positive feedback of Fowler-Nordheim current induces the breakdown across micrometer-scale gap. As the emitted electrons can bridge the gap in the air, the effect of surface accumulated charge was negligibly small across $1 \mu \mathrm{m}$ gap. Figure 14 shows the time dependence of positive ion density at the measuring point shown in Figure 11. The ion density in the vicinity of the cathode increases exponentially with the voltage application. It shows the same tendency to the Fowler-Nordheim current shown in Figure 12.

Figure 15 shows the time dependence of electron density across $3-\mu \mathrm{m}$ gap. At (a) $t_{1}=1 \mathrm{ps}$, the electrons start to be emitted from the cathode and electron density is relatively high in the vicinity of cathode. From (b) $t_{2}=10 \mathrm{ps}$ to (c) $t_{3}=30 \mathrm{ps}$, the emitted electrons move and collide with the insulator surface according to the lines of electric force. At that time, the insulator surface is negatively charged. By (d) $t_{4}=50 \mathrm{ps}$, the electric field is distorted such accumulated charge, and the successive electrons bridged from cathode to anode above the surface of the insulator. The result is consistent to the breakdown path shown in Figure 10; breakdown path was formed above the surface of the insulator.

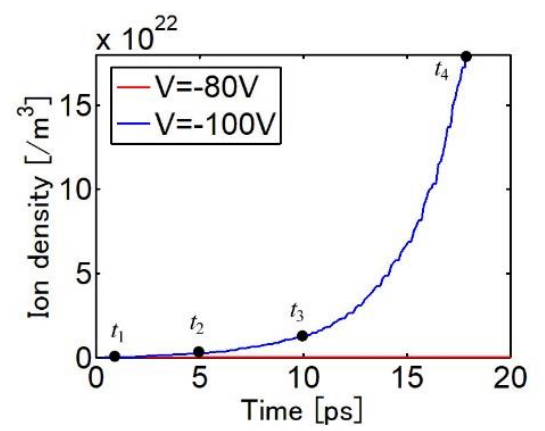

Figure 14. Ion density in the vicinity of the cathode: $1 \mu \mathrm{m}$ gap.

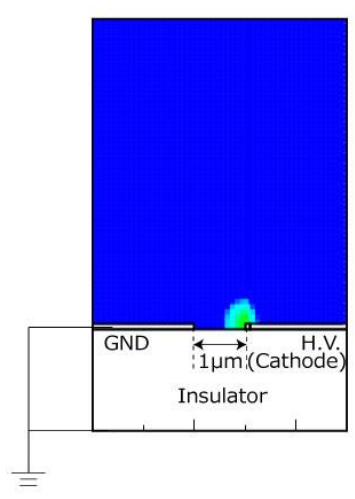

(a)

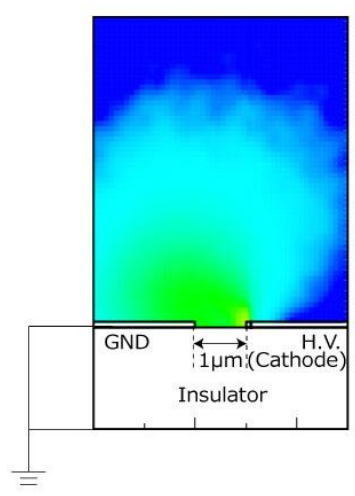

(b)

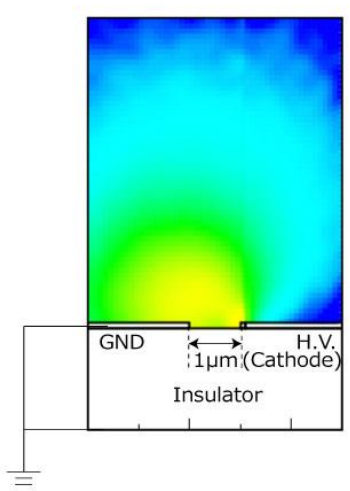

(c)

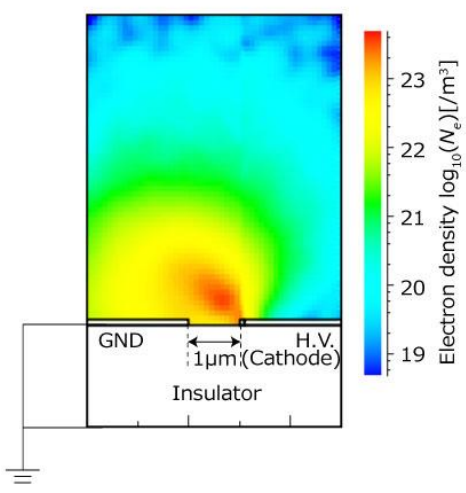

(d)

Figure 13. Time dependent of electron density across $1-\mu \mathrm{m}$ gap. Each figure shows the electron density at (a) $t_{1}=1 \mathrm{ps}$, (b) $t_{2}=5 \mathrm{ps},(\mathrm{c}) t_{3}=10 \mathrm{ps}$, (d) $t_{4}=18$ ps and $t_{1}-t_{4}$ correspond to the black dots shown in Figure 14. 


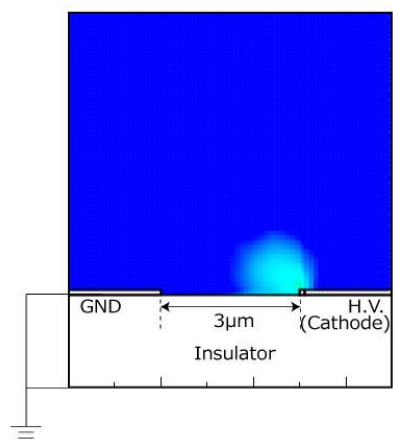

(a)

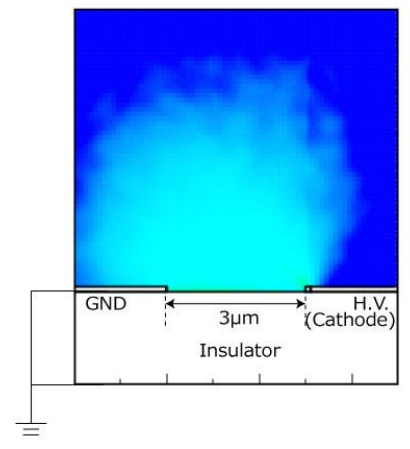

(b)

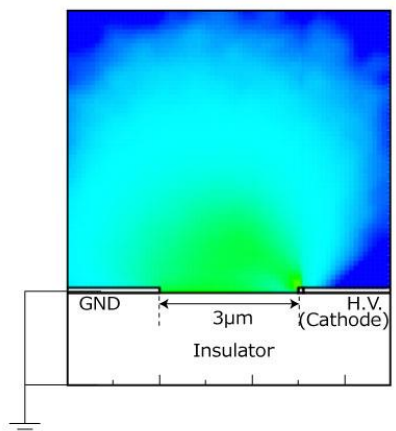

(c)

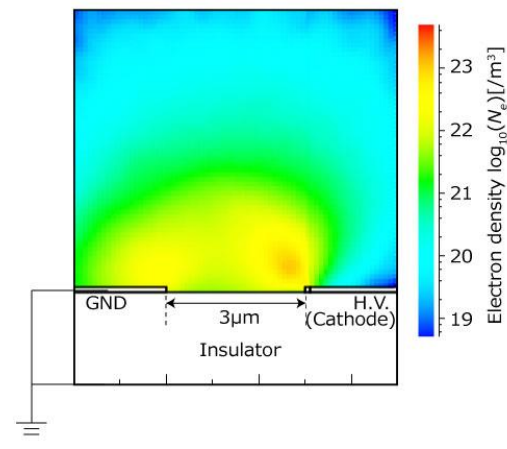

(d)

Figure 15. Time dependent of electron density across $3 \mu \mathrm{m}$ gap. Each figure shows the electron density at (a) $t_{1}=1 \mathrm{ps},(\mathrm{b}) t_{2}=10 \mathrm{ps},(\mathrm{c}) t_{3}=30 \mathrm{ps},(\mathrm{d}) t_{4}=50$ ps and $t_{1}-t_{4}$ correspond to the black dots shown in Figure 16.

Figure 16 shows the time dependence of positive ion density at the measuring point shown in Figure 11. The ion density in the vicinity of the cathode increases exponentially after $t=30$ ps. It shows the same tendency to the Fowler-Nordheim current shown in Figure 12.

Figure 17 shows the time dependence of electron density across $5 \mu \mathrm{m}$ gap. It shows the same tendency to the process across $3 \mu \mathrm{m}$ gap. At the first stage, the emitted electrons move to the insulator surface according to the lines of electric force. The insulator surface is negatively charged and electrons bridged from cathode to anode above the surface of the insulator. Figure 18 shows the time dependence of surface charge distribution across $5 \mu \mathrm{m}$ gap. When $-100 \mathrm{~V}$ is applied, the insulator surface is uniformly charged. It affects the discharge path across the gap. Figure 19 shows the time dependence of positive ion density at the measuring point shown in Figure 11. The ion density in the vicinity of the cathode increases exponentially after $t=80 \mathrm{ps}$. It indicates that it takes $80 \mathrm{ps}$ to charge the insulator surface substantially. It shows the same tendency to the Fowler-Nordheim current shown in Figure 12.

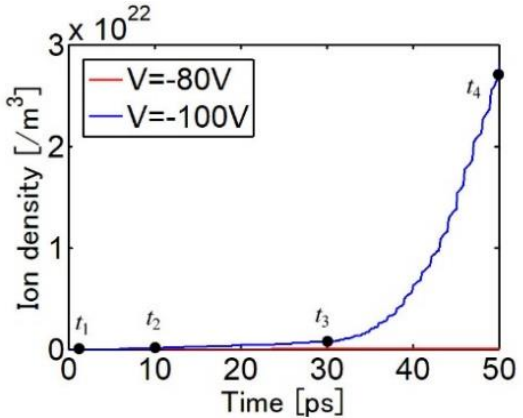

Figure 16. Ion density in the vicinity of the cathode: $3 \mu \mathrm{m}$ gap.

Figure 20 shows the time dependence of electron density under $-350 \mathrm{~V}$ was applied. Considering the long voltage application, $\beta$ was set at 10 . The discharge process shows the same tendency to the one shown in Figure 17; electrons bridged from cathode to anode above the surface of the insulator.

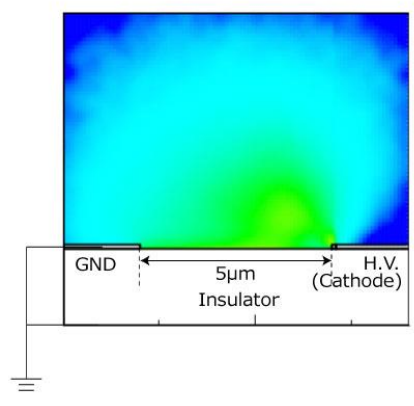

(a)

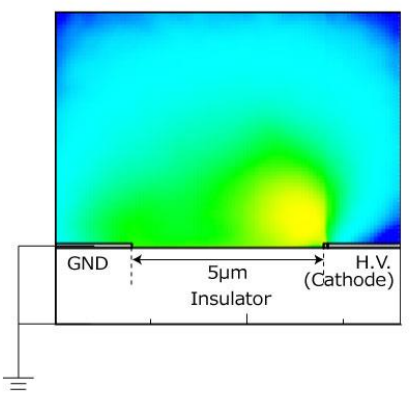

(b)

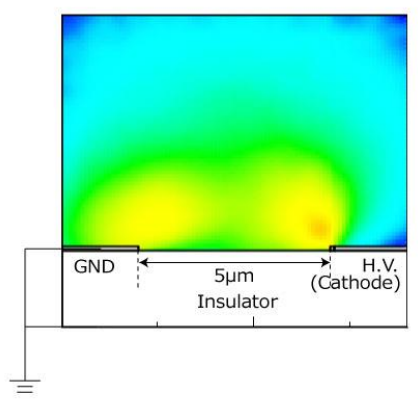

(c)

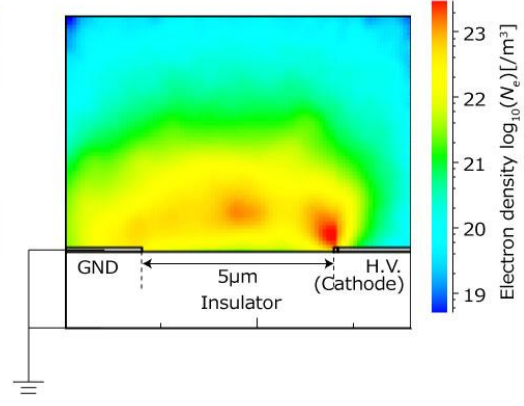

(d)

Figure 17. Time dependent of electron density across $5 \mu \mathrm{m}$ gap. Each figure shows the electron density at (a) $t_{1}=40 \mathrm{ps}$, (b) $t_{2}=60 \mathrm{ps}$, (c) $t_{3}=80 \mathrm{ps,} \mathrm{(d)} t_{4}=$ $100 \mathrm{ps}$ and $t_{1}-t_{4}$ correspond to the black dots shown in Figure 19. 


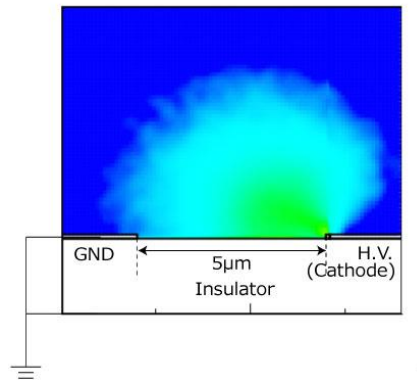

(a)

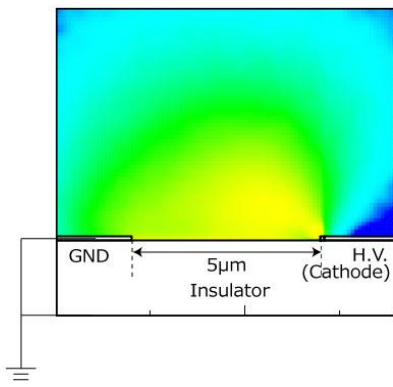

(b)

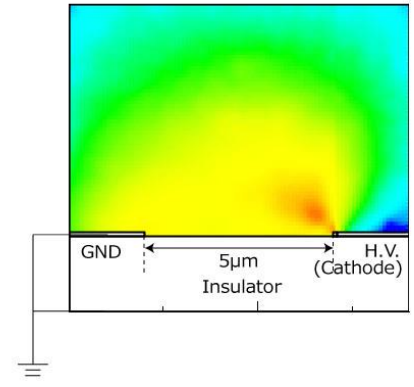

(c)

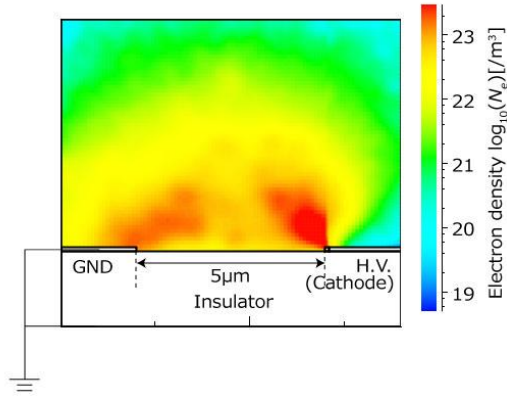

(d)

Figure 20. Time dependent of electron density across $5 \mu \mathrm{m}$ gap under application of $-350 \mathrm{~V}$. Each figure shows the electron density at (a) $t_{1}=2 \mathrm{ps}$, (b) $t_{2}=4.5$ ps, (c) $t_{3}=6.5 \mathrm{ps}$, (d) $t_{4}=8.5 \mathrm{ps}$. The discharge process shows the same tendency to the one shown in Figure 17.

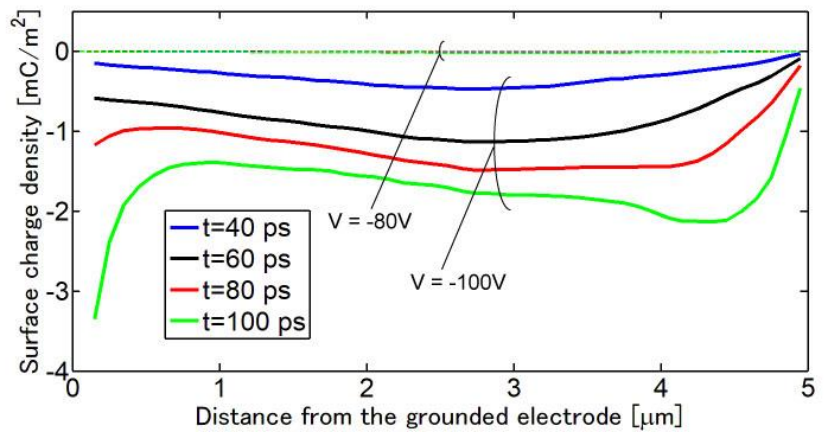

Figure 18. Surface charge density on the insulator surface. When $-100 \mathrm{~V}$ was applied, the surface charge density was higher than the one under $-80 \mathrm{~V}$ was applied all over the insulator surface. The surface charge affected the discharge path and the path was formed above the surface of the insulator.

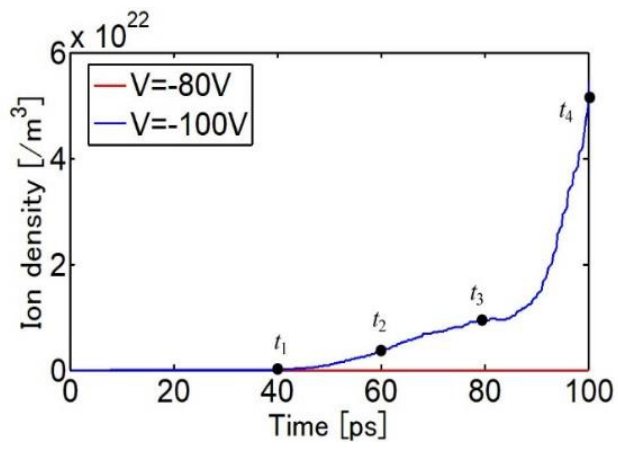

Figure 19. Ion density in the vicinity of the cathode: $5 \mu \mathrm{m}$ gap.

\section{DISCUSSION}

The breakdown process under the negative voltage application is shown in Figure 21. Under the negative voltage application, the initial electrons are provided from the cathode surface (1). As the electric field intensity on the cathode is over $10^{8} \mathrm{~V} / \mathrm{m}$, the electric field emission can occur from the small projection on the cathode. The emitted electrons from the cathode can collide with the neutral particles and the positive space charges are generated in the vicinity of the cathode (2). Consequently, the electric field intensity on the cathode is strengthened by the positive charges in the gas. Therefore, the electric field emission from the cathode is sustained and the discharge path is formed from small projections on the cathode to the anode. When the gap width is larger than $3 \mu \mathrm{m}$, the electrons emitted from small projections on the cathode may collide to $\mathrm{SiO}_{2}$ surface and the surface is negatively charged (3). The surface charge affects the flow of electrons and the discharge path was lifted up over the surface of the insulator (4).

(1)

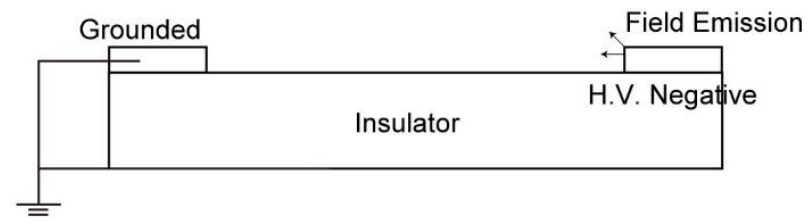

(2)

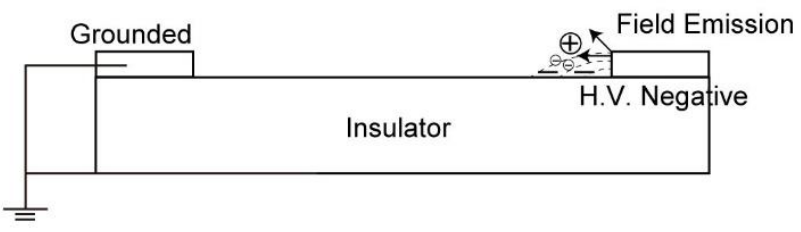

(3)

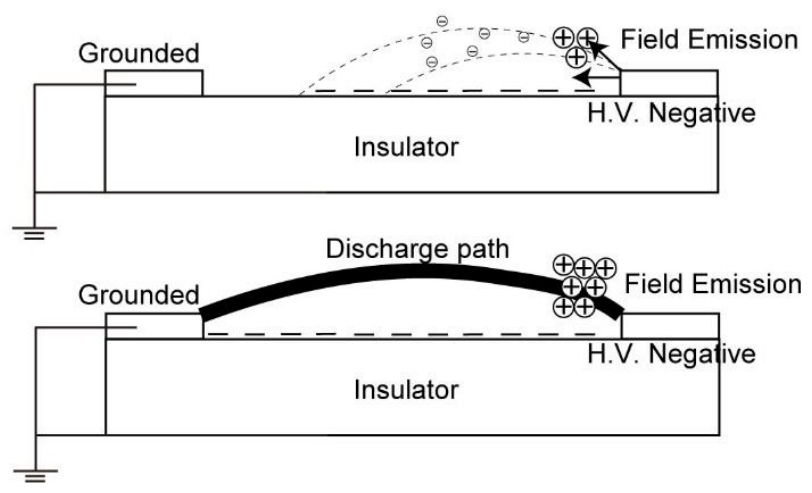

Figure 21. Breakdown process across micrometer-scale gap especially larger than $3 \mu \mathrm{m}$. (1) The initial electrons are provided from the cathode surface by field emission. (2)(3) The emitted electrons collide to $\mathrm{SiO}_{2}$ surface and the surface is negatively charged. The positive space charges are generated in the vicinity of cathode. (4) The discharge path was lifted up over the surface of the insulator by the accumulated surface charges. 


\section{CONCLUSION}

This paper reported breakdown process across micrometerscale gap representing the actual electronic devices. The focus of this research is the micrometer-scale gap including the insulator surface. The electric field distribution in the gap is inhomogeneous and locally high in the vicinity of cathode surface. Experimental and simulated results show that breakdown voltage under negative impulse voltage application is mainly determined by the instantaneous voltage on the cathode surface. It can be explained by the positive feedback process of field emission current from cathode. Field emission current is increased by positive ions generated by collision of electrons to neutral particles. When $-100 \mathrm{~V}$ was applied across several-micrometer surface gap, such feedback process was induced and field emission current increased drastically. Charges were accumulated on the insulator surface by field emission current and the discharge path was lifted up from the insulator surface. At last, the discharge path was formed above the surface of the insulator 100 ps after the voltage was applied.

\section{ACKNOWLEDGMENT}

This work was supported by JSPS KAKENHI Grant Number JP15H06222.

\section{REFERENCES}

[1] J. W. Han, D. I. Moon and M. Meyyappan, "Nanoscale Vacuum Channel Transistor," Nano Letters vol. 17, pp. 2146-2151, 2017.

[2] T. Higuchi et al, "A nanoscale vacuum-tube diode triggered by few-cycle laser pulses,” Appl. Phys. Lett., vol. 106, 051109, 2015.

[3] C. H. Chen et al, "Electrical breakdown phenomena for devices with micron separations," J. Micromech. Microeng. 16, pp. 1366-1373, 2006.

[4] P. Slade and E. Taylor, "Electrical breakdown in atmospheric air between closely spaced $(0.2 \mu \mathrm{m}-40 \mu \mathrm{m})$ electrical contacts," 47th IEEE Holm Conference on Electrical Contacts, 2001, pp. 245-250.

[5] J. M. Torres and R. S. Dhariwal, "Electric field breakdown at micrometer separations in air and vacuum," Microsystem Technologies vol. 6, No. 1, pp. 6-10, 1999.

[6] T. Ono, D. Sim and M. Esashi, "Micro-discharge and electric breakdown in a microgap," J. Micromech. Microeng. 10, pp. 445-451, 2000.

[7] R. C. Sterling et al, "Increased surface flashover voltage in microfabricated devices," Appl. Phys. Lett., 103, 143504, 2013.

[8] G. Meng et al, "Electrical Characteristics of Nanometer Gaps in Vacuum under Direct Voltage," IEEE Trans. Dielectr. Electr. Insul., vol. 21, no. 4, pp. 1950-1956, 2014.

[9] M. Klas et al, "The breakdown voltage characteristics and the secondary electron production in direct current hydrogen discharges for the gaps ranging from $1 \mu \mathrm{m}$ to $100 \mu \mathrm{m}$,” Phys. Lett. A, 376, pp. 1048-1052, 2012.

[10] M. Radmilović-Radjenović et al, "Particle-in-cell simulation of gas breakdown in microgaps," J. Phys. D, vol. 38, pp. 950-954, 2005.

[11] A. Venkattraman et al, "Direct measurements and numerical simulations of gas charging in microelectromechanical system capacitive switches," Appl. Phys. Lett., 100, 083503, 2012.

[12] D. B. Go and D. A. Pohlman, "A mathematical model of the modified Paschen's curve for breakdown in microscale gaps," J. Appl. Phys., 107, 103303, 2010.

[13] G. Meng et al, "Effect of electrode geometry on the vacuum breakdown behaviors at nanoscale," IEEE Int. Conf. Dielectr. (ICD),2016, pp. 1925.

[14] C. Men et al, "Influence of crystal morphology on breakdown characteristics in vacuum between nano scale gaps," Int. Conf. Cond. Monitoring Diagnosis (CMD), 2016, F1-4.

[15] G. Meng et al., "Demonstration of field emission driven microscale gas breakdown for pulsed voltages using in-situ optical imaging," Physics of Plasmas, vol. 25, 082116, 2018.

[16] H. Iwabuchi et al, "Pre-breakdown Phenomenon in Micrometer-scale Gap," 31st Int. Conf. Plasma in Ionized Gases, 2013, pp 2-13.
[17] H. Iwabuchi et al, "Breakdown Characteristics across Micrometer-scale Surface Gap with Aluminum Electrodes," IEEJ Trans. FM, vol. 134, No. 8, pp. 485-486, 2014.

[18] R. H. Fowler and L. Nordheim, "Electron Emission in Intense Electric Fields," Proc. R. Soc; London A 119, 173, 1928.

[19] Y. Itikawa, "Cross Sections for Electron Collisions with Nitrogen Molecules," J. Phys. Chem. Ref. Data, 35, 31, 2006.

[20] R. R. Laher and F. R. Gilmore, "Updated Excitation and Ionization Cross Sections for Electron Impact on Atomic Oxygen," J. Phys. Chem. Ref. Data 19, 277, 1990.

[21] J. J. Fijol et al, "Secondary electron yield of $\mathrm{SiO}_{2}$ and $\mathrm{Si}_{3} \mathrm{~N}_{4}$ thin films for continuous dynode electron multipliers," Appl. Surface Sci., vol. 4849, pp 464-471, 1991.

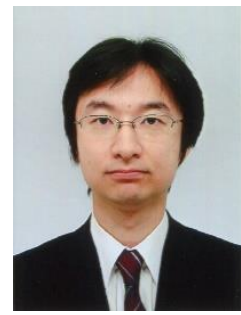

Hiroyuki Iwabuchi (M'15) received his B.S., M.S., and Dr. Eng. degrees in electrical engineering from the University of Tokyo, Tokyo, Japan, in 2010, 2012, and 2015, respectively. Since April 2015, he has been working as an assistant professor in Yokohama National University. He is engaged in the research on the current interruption and the numerical simulation of breakdown phenomena.

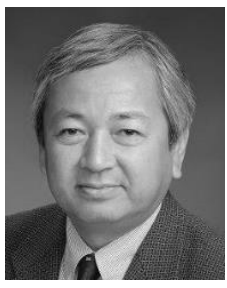

Tsutomu Oyama (S'78-M'84) received his B.S., M.S., and Dr. Eng. degrees in electrical engineering from the University of Tokyo, Tokyo, Japan, in 1978, 1980, and 1983, respectively. Since 1983, he has been with Yokohama National University, Japan, where he is currently a Professor. His research interests include analysis, operation, and planning of power systems. Prof. Oyama is a distinguished member of CIGRE, a member of Japan Society of Energy and Resources, and a senior member of IEE of Japan.

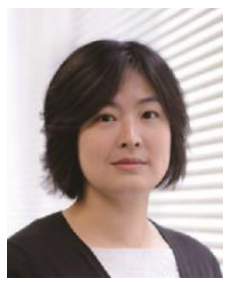

Akiko Kumada (M'03) received the B. Eng., M. Eng., and Dr. degrees from the University of Tokyo in 1994, 1996, and 1999, respectively. After working as a research associate in the same university from 1999 to 2001, she joined the Tokyo Electric Power Company as a researcher from 2001 to 2003. Since April 2003, she has been in the University of Tokyo and is now a professor of electrical engineering.

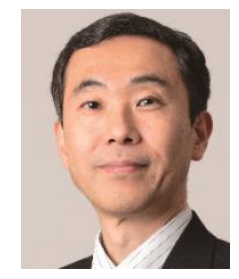

Kunihiko Hidaka (M'76-SM'04-F'12) received the B.E., M.E., and D.Eng. degrees from the University of Tokyo in 1976, 1978, and 1981 respectively. Since 1987 he has been with the department of electrical engineering of the University of Tokyo. He has been engaged in research on electrical breakdown phenomena concerned with high voltage technology, and his work has won premiums and awards from both the Japanese and British IEE. He is a Fellow of IEEE and IEEJ. 